\title{
Pulmonary toxicity in stage III non-small cell lung cancer patients treated with high dose (74 Gy) 3-dimensional conformal thoracic radiotherapy and concurrent chemotherapy following induction chemotherapy: A secondary analysis of Cancer and Leukemia Group B (CALGB) trial 30105
}

\author{
Joseph K. Salama, MD ${ }^{5}$, Thomas E. Stinchcombe, MD², Lin Gu, MS ${ }^{3}$, Xiaofei Wang, Ph.D ${ }^{3}$, \\ Karen Morano, RT (T) CMD ${ }^{7}$, Jeffrey A. Bogart, MD ${ }^{4}$, Jeffrey C. Crawford, MD ${ }^{5}$, Mark A. \\ Socinski, MD $^{2}$, A. William Blackstock, MD $^{6}$, and Everett E. Vokes, MD $^{1}$ on behalf of For the \\ Cancer and Leukemia Group B \\ ${ }^{1}$ University of Chicago, Chicago, IL, supported by CA41287 \\ ${ }^{2}$ University of North Carolina at Chapel Hill, Chapel Hill, NC, supported by CA47559 \\ ${ }^{3}$ CALGB Statistical Center, Duke University Medical Center, Durham, NC, supported by CA33601 \\ ${ }^{4}$ State University of New York Upstate Medical University, Syracuse, NY, supported by CA21060 \\ ${ }^{5}$ Duke University Medical Center, Durham, NC, supported by CA47577 \\ ${ }^{6}$ Wake Forest University School of Medicine, Winston-Salem, NC, supported by CA03927 \\ ${ }^{7}$ Quality Assurance Review Center, Lincoln, RI, supported by CA29511
}

\begin{abstract}
Purpose-CALGB 30105 tested two different concurrent chemoradiotherapy platforms with high dose (74 Gy) 3-D conformal radiotherapy (3DCRT) following two cycles of induction chemotherapy for stage IIIA/IIIB NSCLC patients to determine if either could achieve a primary endpoint of > 18 month median survival. Final results of 30105 demonstrated that induction
\end{abstract}

\footnotetext{
(C) 2011 Elsevier Inc. All rights reserved.

Address for Correspondence: Joseph K. Salama, M.D., Department of Radiation Oncology, Box 3085, Duke University Medical Center, Ph: 919.668.7336, Fax: 919.668.7345, joseph.salama@ duke.edu.

Publisher's Disclaimer: This is a PDF file of an unedited manuscript that has been accepted for publication. As a service to our customers we are providing this early version of the manuscript. The manuscript will undergo copyediting, typesetting, and review of the resulting proof before it is published in its final citable form. Please note that during the production process errors may be discovered which could affect the content, and all legal disclaimers that apply to the journal pertain.

The following institutions participated in this study:

Christiana Care Health Services, Inc. CCOP, Wilmington, DE-Stephen Grubbs, M.D.., supported by CA45418 Duke University Medical Center, Durham, NC-Jeffrey Crawford, M.D., supported by CA47577

Kansas City Community Clinical Oncology Program CCOP, Kansas City, MO-Jorge C. Paradelo, M.D.

Roswell Park Cancer Institute, Buffalo, NY-Ellis Levine, M.D., supported by CA02599

University of California at San Diego, San Diego, CA-Joanne Mortimer, M.D., supported by CA11789

University of Minnesota, Minneapolis, MN-Bruce A Peterson, M.D., supported by CA16450

University of Missouri/Ellis Fischel Cancer Center, Columbia, MO-Michael C Perry, M.D., supported by CA12046

Wake Forest University School of Medicine, Winston-Salem, NC-David D Hurd, M.D., supported by CA03927

Southeast Cancer Control Consortium Inc. CCOP, Goldsboro, NC-James N. Atkins, M.D., supported by CA45808

University of North Carolina at Chapel Hill, Chapel Hill, NC-Thomas C. Shea, M.D., supported by CA47559

University of Massachusetts Medical School, Worcester, MA-William V. Walsh, M.D., supported by CA37135

Conflict of Interest : No conflicts of interest were reported.
} 
carboplatin and gemcitabine and concurrent gemcitabine 3DCRT was not feasible due to treatment related toxicity. However, induction and concurrent carboplatin/paclitaxel with 74 Gy 3DCRT had a median survival of 24 months, and is the basis for the experimental arm in CALGB 30610/ RTOG 0617/N0628. We conducted a secondary analysis of all patients to determine predictors of treatment related pulmonary toxicity.

Methods and Materials-Patient, tumor, and treatment related variables were analyzed to determine their relation with treatment related pulmonary toxicity.

Results-Older age, higher N stage, larger PTV1, smaller TLV/PTV1 ratio, larger V20, and larger mean lung dose were associated with increasing pulmonary toxicity on univariate analysis. Multivariate analysis confirmed that V20 and nodal stage as well as treatment with concurrent gemcitabine were associated with treatment related toxicity. A high risk group comprising patients with N3 disease and V20>38\% was associated with $80 \%$ of grade 3-5 pulmonary toxicity cases.

Conclusions-Elevated V20 and N3 disease status are important predictors of treatment related pulmonary toxicity in patients treated with high dose 3DCRT with concurrent chemotherapy. Further studies may use these metrics in considering patients for these treatments.

\section{Keywords}

Chemoradiotherapy; 3D Conformal Radiotherapy; Non-small cell lung cancer; pulmonary toxicity

\section{Introduction}

Lung cancer remains the leading cause of cancer mortality. Approximately $85 \%$ of lung cancer patients have non-small cell (NSCLC) histology, and one-third of NSCLC patients present with stage IIIA or IIIB disease. For patients with preserved performance status and adequate organ function, the combination of chemotherapy and radiation therapy is the standard of care $(1,2)$. Concurrent chemoradiotherapy results in improved survival compared to sequential chemotherapy and radiation.(3)

The development of three-dimensional conformal radiotherapy (3DCRT) planning techniques has led to improved radiation delivery facilitating better tumor coverage, compared to conventional techniques, while minimizing exposure of surrounding normal tissues.(4-7) The ability for 3DCRT to decrease normal organ radiation exposure led several investigators to perform phase I and II trials of escalated dose 3DCRT either alone or in combination with chemotherapy in NSCLC.(8-15)

Cancer and Leukemia Group B (CALGB) 30105 was a two arm randomized phase II trial investigating induction and concurrent chemotherapy with 3DCRT to 74 Gy. Arm A investigated induction and concurrent chemotherapy with carboplatin and paclitaxel, and arm B investigated induction chemotherapy with carboplatin and gemcitabine followed by single agent concurrent gemcitabine and 3-D CRT. (16) Arm B was closed prematurely due to a high rate of grade 4 to 5 pulmonary toxicity. We performed this secondary analysis to investigate the correlation between baseline pulmonary function and radiation treatment planning parameters as risk factors for pulmonary toxicity in patients treated with concurrent chemotherapy and 74 Gy 3DCRT.

\section{Patients and Methods}

\section{Eligibility}

CALGB 30105 eligibility criteria have been published previously.(16) Briefly, patients with histologically or cytologically confirmed stage IIIA-IIIB (AJCC 2000) unresectable 
NSCLC, Eastern Cooperative Oncology Group (ECOG) performance status (PS) of 0-1, and normal organ and marrow function were eligible. Patients with direct invasion of the vertebral bodies or scalene, supracalvicular, or contralateral hilar adenopathy were ineligible. All patients were required to have a forced expiratory volume in 1 second (FEV-1) of $>1.2$ L. Following informed consent patients were randomized to treatment arm A or B (Figure 1). The trial was approved by the institutional review boards of the participating institutions.

\section{Chemotherapy treatment plan}

Patients in arm A received induction chemotherapy with carboplatin area under the curve (AUC) of 6 using the Calvert equation(17) and paclitaxel $225 \mathrm{mg} / \mathrm{m}^{2}$ on days 1 and 22 . On day 43 patients received weekly carboplatin AUC $=2$ and paclitaxel $45 \mathrm{mg} / \mathrm{m}^{2}$ for seven weeks concurrent with 3DCRT. Patients in arm B received induction chemotherapy carboplatin AUC $=5$ using the Calvert equation on days 1 and 22, and gemcitabine $1000 \mathrm{mg} /$ $\mathrm{m}^{2}$ on days $1,8,22$, and 29 . On day 43 patients received twice weekly gemcitabine $35 \mathrm{mg} /$ $\mathrm{m}^{2}$ for seven weeks concurrent with 3DCRT. Details of premedication, dose modifications, and chemotherapy treatment delays have been published previously.(16)

\section{Radiation treatment plan}

Prior to induction chemotherapy, all patients underwent contrast enhanced computed tomography (CT) based radiation treatment planning in customized immobilization devices. For the first phase of treatment, the primary tumor and pathologically involved adenopathy (those with a necrotic center, biopsy proven, FDG-positron emission tomography (PET) avid, or measuring $>1 \mathrm{~cm}$ in short axis diameter) were contoured on each slice of the planning CT as gross tumor volume (GTV1). Clinical target volume 1 (CTV1) was created by expanding GTV1 by $2 \mathrm{~cm}$ in all directions except for the interface of the primary tumor and normal lung parenchyma where it was expanded $0.5 \mathrm{~cm}$ or more at the discretion of the treating radiation oncologist. Additionally, elective treatment of ipsilateral upper paratracheal and contralateral lower paratracheal nodal stations for T2N2 patients or lower paratracheal and subcarinal regions for T3N1 patients could be included in CTV1. Planning target volume (PTV1) was created by expanding CTV1 by $1 \mathrm{~cm}$ in all directions. For the second phase of treatment, at the discretion of the treating radiation oncologist, GTV2 could be redefined as the reduced GTV volume following induction chemotherapy. For patients not responding to induction chemotherapy GTV2 was identical to GTV1. CTV2 and PTV2 were created by sequential expansions similar to the first course. For both courses, the lungs, heart, and spinal cord were contoured on each planning CT slice.

3DCRT was required for this study. Beam arrangements and treatment portals were chosen to maximize tumor coverage and minimize normal tissue exposure. Photon beam energies of $4 \mathrm{MV}$ or higher were required. The prescription radiation dose for the first course was $40 \mathrm{~Gy}$ in 2 Gy daily fractions to PTV1, followed by 34 Gy in 2 Gy daily fractions to PTV2.

Radiation dose was prescribed to isocenter and accounted for tissue heterogeneity. Radiation planning required that $100 \%$ of the PTV be encompassed by the $95 \%$ isodose surface and no more than $10 \%$ of the volume receives more than $110 \%$ of the prescription dose. The protocol mandated that the maximum dose to the spinal cord be $49 \mathrm{~Gy}$, and wherever possible, without shielding gross tumor, the dose to the lung parenchyma, esophagus, and heart should be minimized. No specific dose-volume constraints were placed on the lung, heart, and esophagus. Prior to the start of 3DCRT radiation plans were required to be reviewed by the Quality Assurance Review Center (QARC) where an approved 3-D benchmark was on file. 


\section{Statistical Analysis Method}

CALGB statisticians performed all statistical analyses. All toxicity was coded using the Common Toxicity Criteria version 2.0 which was current at the time of the study. This scoring system included the use of the RTOG/EORTC late radiation morbidity scoring system-LUNG for toxicities greater than 90 days following the completion of radiation (Supplemental Table 1). Radiation planning variables analyzed for an association with pulmonary toxicity included the volume of tumor free lung receiving $\geq 5,10$, and $20 \mathrm{~Gy}$, (Lung V5,V10, and V20), the total lung volume (TLV), mean lung dose (MLD), maximum lung dose, pre-induction gross tumor volume (GTV1), PTV1, the ratios of PTV1 and GTV1 to the TLV, radiation energy, number of radiation beams used, and radiation field size, measured as the equivalent square of the largest field. Pretreatment pulmonary function was assessed via FEV-1 (the only pulmonary function parameter collected for the study). Other variables analyzed included age, $\mathrm{T}$ stage, $\mathrm{N}$ stage, and maximum tumor size.

Univariate analyses were performed using Fisher's exact 2-sided test for categorical variables and Wilcoxon 2-sided test on continuous variables to examine the relationship between maximum pulmonary toxicity (grade $0-2$ vs. $3-5$ ) and patient and treatment related factors. A multivariate logistic regression model was then created including significant variables from the univariate analyses as well as treatment $\operatorname{arm}(\mathrm{A}$ or B).

\section{Results}

\section{Patient characteristics}

Between March 2002 and November 2004, 69 patients were enrolled with 68 eligible, 42 on $\operatorname{arm}$ A, and 26 on arm B (Figure 1). Patient and tumor characteristics are summarized in Table 1 . The median age was 61 years (range, 38 to 79 years), and two most common histologies were adenocarcinoma (35\%) and squamous carcinoma (35\%). The median FEV-1 was $2.08 \mathrm{~L}$ (range, 1.24 to $3.73 \mathrm{~L}$ ). Characteristics were well balanced between the two arms.

\section{Analysis of Patient, Tumor, and Treatment Characteristics Associated with Toxicity}

Grade 3 or higher pulmonary toxicity was seen in a total of $12(18 \%)$ patients, five patients (12\%) in arm A and seven patients (27\%) in Arm B (Table 2); no patients in arm A and two patients $(8 \%)$ in arm B experienced grade 5 pulmonary toxicity (supplemental Table 2 and Supplemental Figure 1). Assessment of patient, tumor, and radiation planning parameters in patients who experienced $\geq$ grade 3 pulmonary toxicity revealed that older age $(\mathrm{p}=0.0047)$, higher N stage $(\mathrm{p}=0.0343)$, larger PTV1 ( $\mathrm{p}=0.0946)$, smaller TLV/PTV1 ratio $(\mathrm{p}=0.0639)$, larger V20 ( $\mathrm{p}=0.0168)$ and larger mean lung dose $(\mathrm{p}=0.0973)$ were associated with an increased risk of pulmonary toxicity. T stage, maximum tumor size, FEV1, maximum lung dose, total lung volume, V5, V10, and radiation field size were not significantly associated with treatment related pulmonary toxicity as shown in Table 3 . These results were similar whether toxicity was grouped as grade $0-2,3$, and $4-5$ or as grade $0-2$ and $3-5$.

Multivariate analysis was performed to evaluate the relationship between pulmonary toxicity and radiotherapy related factors. Some variables identified on univariate testing, were excluded from this model due to missing values in patients who experienced grade 3-5 pulmonary toxicity including: maximum tumor size, PTV-1, and MLD. Additionally, V5 and V10 were excluded from the analysis, as more V20 values were available and V20 was highly correlated with both V5 and V10. Variables included in the analysis were: age, sex, T stage, N stage, FEV1, GTV1, TLV, TLV/GTV1, V20, tumor location (left vs. right), radiation energy, number of fields, and radiation field size. This model revealed that a 
smaller V20 (OR 0.8 (95\% CI: 0.7-1, p=0.0414), lower N stage, and treatment on arm A were associated with decreased risk of grade 3-5 pulmonary toxicity as shown in Table 4 .

To test whether patients with missing data were more likely to experience pulmonary toxicity, an indicator variable was created to denote whether at least one of the identified predictors of pulmonary toxicity (V20, PTV1, MLD, GTV1, TLV, and maximum lung dose) was missing. This was then fit to a regression model. This model revealed no association between a missing variable and pulmonary toxicity [OR: 0.32 (95\% CI: $0.07-$ $1.55(\mathrm{p}=0.1558)]$. Therefore, the missing data did not seem to skew the analysis.

We then carefully analyzed the parameters to determine if binary values could be used to predict toxicity. We found that the median V20 in patients experiencing grade 0-2 pulmonary toxicity was $32 \%$ compared to $40 \%$ in those experiencing grade $3-5$ pulmonary toxicity. The median lung dose of patients experiencing grade $0-2$ pulmonary toxicity was 18.5 Gy compared to 21.5 Gy in patients experiencing grade 3-5 pulmonary toxicity. The median target volume (PTV1) associated with grade 4-5 pulmonary toxicity was $1688 \mathrm{cc}$. The median target volume associated with grade $0-2$ or grade 3 toxicity was $772 \mathrm{cc}$ or 725 cc, respectively. Additionally, a small TLV/PTV1 ratio (median 1.5) was associated with grade 4-5 toxicity compared to median 29.7 and 32.1 for grades $0-2$ and 3, respectively. We subsequently entered the above binary predictors into a multivariate model. This reduced logistic regression model showed that patients with V20 greater than $38 \%$ were more likely to have grade 3-5 pulmonary toxicity as were those with $\mathrm{N} 3$ disease and those treated on arm B.

Finally, using the parameters above, we classified patients as high risk if they were N3 or had a V20 $\geq 38 \%$, or low risk if they were N0-2 and had a V20<38\%. Using Fisher's Exact Test, we found that risk group was highly correlated with treatment related toxicity $(\mathrm{p}=0.0313)$ (Table 5). Using this classification schema correctly identified 80\% (95\% CI: $44-97)$ of patients with grade $3-5$ toxicity and $62 \%$ (95\% CI: 46-76) of patients with grade $0-2$ toxicity This classification was independent of progression locoregionally or distantly (data not shown).

\section{Discussion}

We conducted this secondary analysis of patients treated on CALGB 30105 to determine predictive factors for pulmonary toxicity following concurrent chemotherapy and high dose (74 Gy) 3DCRT in unresectable stage III NSCLC patients. This group of patients was useful to evaluate as all were treated prospectively on protocol with pretreatment quality assurance review. In this analysis we found that previously described predictors of treatment related pulmonary toxicity in patients treated to standard dose (60 Gy) 3DCRT and concurrent chemotherapy (MLD and V20) were also predictive for toxicity in patients treated with high dose (74 Gy) 3DCRT with concurrent chemotherapy. Previous analyses of patients treated primarily with radiotherapy alone $(5,18)$ and concurrent chemotherapy and radiation therapy (19) have revealed that higher V20 and mean lung dose were both strong predictors of treatment related pulmonary toxicity. We also identified a trend for increased risk of toxicity with increasing V5 and V10, as others have described. In our series, these did not reach statistical significance, but were clearly correlated with V20, and lack of significance may have been due to small sample size.

We identified that patients treated to larger PTV volumes as well as those with N3 disease were at risk for increased treatment related pulmonary toxicity. This likely correlates increasing toxicity with increasing volume of tumor free lung irradiated which is common with contralateral nodal spread. Furthermore, patients who had large target volumes 
compared to their total lung volume were more likely to have grade 4-5 pulmonary toxicity. Although not mandated in the protocol, patients in this study were often treated to an initial large elective volume including bilateral paratracheal, subcarinal, and ipsilateral hilar irradiation, which contributed to irradiation of large volumes of tumor free lung. Ongoing CALGB combined modality studies for stage IIIA-IIIB non-small cell lung cancer no longer treat nodal regions electively, and should have smaller PTV volumes exposing less tumor free lung to radiation.

These data agree with a prior study that identified patients with larger GTVs and smaller lung volumes being associated with higher risk of toxicity(19) although others have not confirmed these findings.(5) Furthermore, larger GTVs and PTVs have been associated with worse overall survival in patients treated with radiation alone (20-22) as well as with concurrent chemotherapy and radiotherapy. (23) These findings make logical sense, as irradiating larger lung volumes exposes more alveoli to the effects of radiotherapy, and larger tumors may be associated with a reduced rate of local control and/or a higher rate of occult distant metastases.

This analysis confirmed that treatment arm was associated with higher risk of treatment related pulmonary toxicity. Although more patients receiving concurrent radiotherapy and gemcitabine had radiation treatment parameters associated with increasing pulmonary toxicity, multivariate analyses identified that treatment regimen was independently associated with increasing pulmonary toxicity risk. Arrieta et al also found that concurrent radiotherapy and weekly gemcitabine $\left(200 \mathrm{mg} / \mathrm{m}^{2}\right)$ following carboplatin AUC of 2.5 and gemcitabine $\left(800 \mathrm{mg} / \mathrm{m}^{2}\right)$ on days 1 and 8 every 21 days for two cycles, was associated with $31.6 \%$ rate of grade $3-5$ pneumonitis. (24)Therefore, the radiosensitizing properties of gemcitabine on normal tissues may be too pronounced, especially in the dose escalated 3DCRT setting. Excess toxicity has been seen with concurrent paclitaxel $30 \mathrm{mg} / \mathrm{m}^{2}$ and gemcitabine $\left(300 \mathrm{mg} / \mathrm{m}^{2}\right.$ weekly regimens in RTOG 0017 (25), however the gemcitabine $300 \mathrm{mg} / \mathrm{m}^{2}$ and carboplatin AUC $=2$ weekly arm was found to be tolerable. Grade 3 pulmonary toxicity was observed in 7 of 23 patients treated in the pilot trial of thoracic radiation and concurrent twice weekly gemcitabine.(26) Therefore, the radiosensitizing properties of gemcitabine may have a narrow therapeutic window especially in the setting of dose escalated 3-D CRT.

The results of CALGB 30105 contributed significantly to the design of the experimental arm (74 Gy 3DCRT with concurrent carboplatin/paclitaxel +/- cetuximab) in the ongoing phase III intergroup study (RTOG 0617/CALGB 30609/NCCTG N0628/ECOG R0617). The findings of this analysis will be immediately useful to assist in the selection of a population that has a higher probability of tolerating, and potentially benefiting from, high dose 3DCRT and concurrent chemotherapy. Furthermore, the planning parameters identified may help to refine the criteria used to evaluate and select appropriate treatment plans for patients receiving concurrent chemotherapy and high dose 3DCRT.

As with any retrospective analysis, these data should be viewed cautiously. However, they are supported by other series in the literature and may serve to refine guidelines for future non-small cell lung cancer combined modality studies. This is even more important as radiotherapy planning and delivery technologies, such as respiratory motion tracking and daily patient imaging, have evolved, improving the ability to further limit the exposure of tumor free lung.

\section{Supplementary Material}

Refer to Web version on PubMed Central for supplementary material. 


\section{Acknowledgments}

The research for CALGB 30105 was supported, in part, by grants from the National Cancer Institute (CA31946) to the Cancer and Leukemia Group B (Monica M. Bertagnolli, MD, Chairman), the CALGB Statistical Center (Daniel Sargent, PhD, CA33601), and to the Quality Assurance Review Center (QARC) CA29511 (T.J. Fitzgerald MD, Director). The content of this manuscript is solely the responsibility of the authors and does not necessarily represent the official views of the National Cancer Institute.

The authors would also like to acknowledge the assistance of Fran Laurie and Donna Wardle for their assistance at QARC.

\section{References}

1. Pfister DG, Johnson DH, Azzoli CG, et al. American Society of Clinical Oncology Treatment of Unresectable Non-Small Cell Lung Cancer Guideline: Update 2003. J Clin Oncol. 2004; 22:330353. [PubMed: 14691125]

2. Jett JR, Schild SE, Keith RL, et al. Treatment of non-small cell lung cancer, stage IIIB: ACCP evidence-based clinical practice guidelines (2nd edition). Chest. 2007; 132:266S-276S. [PubMed: 17873173]

3. Auperin A, Le Pechoux C, Rolland E, et al. Meta-Analysis of Concomitant Versus Sequential Radiochemotherapy in Locally Advanced Non-Small-Cell Lung Cancer. J Clin Oncol. 28:21812190. [PubMed: 20351327]

4. Yorke ED, Jackson A, Rosenzweig KE, et al. Correlation of dosimetric factors and radiation pneumonitis for non-small-cell lung cancer patients in a recently completed dose escalation study. Int J Radiat Oncol Biol Phys. 2005; 63:672-682. [PubMed: 15939548]

5. Graham MV, Purdy JA, Emami B, et al. Clinical dose-volume histogram analysis for pneumonitis after 3D treatment for non-small cell lung cancer (NSCLC). Int J Radiat Oncol Biol Phys. 1999; 45:323-329. [PubMed: 10487552]

6. Yorke ED, Jackson A, Rosenzweig KE, et al. Dose-volume factors contributing to the incidence of radiation pneumonitis in non-small-cell lung cancer patients treated with three-dimensional conformal radiation therapy. Int J Radiat Oncol Biol Phys. 2002; 54:329-339. [PubMed: 12243805]

7. Claude L, Perol D, Ginestet C, et al. A prospective study on radiation pneumonitis following conformal radiation therapy in non-small-cell lung cancer: clinical and dosimetric factors analysis. Radiother Oncol. 2004; 71:175-181. [PubMed: 15110451]

8. Belderbos JS, De Jaeger K, Heemsbergen WD, et al. First results of a phase I/II dose escalation trial in non-small cell lung cancer using three-dimensional conformal radiotherapy. Radiother Oncol. 2003; 66:119-126. [PubMed: 12648783]

9. Schild SE, McGinnis WL, Graham D, et al. Results of a Phase I trial of concurrent chemotherapy and escalating doses of radiation for unresectable non-small-cell lung cancer. Int J Radiat Oncol Biol Phys. 2006; 65:1106-1111. [PubMed: 16730134]

10. Bradley JD, Graham MV, Winter KW, et al. Acute and late toxicity results of RTOG 9311: a dose escalation study using 3D conformal radiation therapy in patients with inoperable non-small cell lung cancer. Int J Radiat Oncol Biol Phys. 2003; 57:S137-S138.

11. Sim S, Rosenzweig KE, Schindelheim R, et al. Induction chemotherapy plus three-dimensional conformal radiation therapy in the definitive treatment of locally advanced non-small-cell lung cancer. Int J Radiat Oncol Biol Phys. 2001; 51:660-665. [PubMed: 11597806]

12. Wu KL, Jiang GL, Liao Y, et al. Three-dimensional conformal radiation therapy for non-small-cell lung cancer: a phase I/II dose escalation clinical trial. Int J Radiat Oncol Biol Phys. 2003; 57:1336-1344. [PubMed: 14630271]

13. Marks LB, Garst J, Socinski MA, et al. Carboplatin/paclitaxel or carboplatin/vinorelbine followed by accelerated hyperfractionated conformal radiation therapy: report of a prospective phase I dose escalation trial from the Carolina Conformal Therapy Consortium. J Clin Oncol. 2004; 22:43294340. [PubMed: 15514374]

14. Socinski MA, Rosenman JG, Halle J, et al. Dose-escalating conformal thoracic radiation therapy with induction and concurrent carboplatin/paclitaxel in unresectable stage IIIA/B nonsmall cell lung carcinoma: a modified phase I/II trial. Cancer. 2001; 92:1213-1223. [PubMed: 11571735] 
15. Socinski MA, Morris DE, Halle JS, et al. Induction and concurrent chemotherapy with high-dose thoracic conformal radiation therapy in unresectable stage IIIA and IIIB non-small-cell lung cancer: a dose-escalation phase I trial. J Clin Oncol. 2004; 22:4341-4350. [PubMed: 15514375]

16. Socinski MA, Blackstock AW, Bogart JA, et al. Randomized phase II trial of induction chemotherapy followed by concurrent chemotherapy and dose-escalated thoracic conformal radiotherapy (74 Gy) in stage III non-small-cell lung cancer: CALGB 30105. J Clin Oncol. 2008; 26:2457-2463. [PubMed: 18487565]

17. Calvert A, Newell D, Gumbrell L, et al. Carboplatin dosage: Prospective evaluation of a simple formula based on renal function. J Clin Oncol. 1989; 7:1748-1756. [PubMed: 2681557]

18. Kwa SL, Lebesque JV, Theuws JC, et al. Radiation pneumonitis as a function of mean lung dose: an analysis of pooled data of 540 patients. Int J Radiat Oncol Biol Phys. 1998; 42:1-9. [PubMed: 9747813]

19. Wang S, Liao Z, Wei X, et al. Analysis of clinical and dosimetric factors associated with treatment-related pneumonitis (TRP) in patients with non-small-cell lung cancer (NSCLC) treated with concurrent chemotherapy and three-dimensional conformal radiotherapy (3D-CRT). Int J Radiat Oncol Biol Phys. 2006; 66:1399-1407. [PubMed: 16997503]

20. Bradley JD, Leumwananonthachai N, Purdy JA, et al. Gross tumor volume, critical prognostic factor in patients treated with three-dimensional conformal radiation therapy for non-small-cell lung carcinoma. Int J Radiat Oncol Biol Phys. 2002; 52:49-57. [PubMed: 11777621]

21. Werner-Wasik M, Swann RS, Bradley J, et al. Increasing tumor volume is predictive of poor overall and progression-free survival: secondary analysis of the Radiation Therapy Oncology Group 93-11 phase I-II radiation dose-escalation study in patients with inoperable non-small-cell lung cancer. Int J Radiat Oncol Biol Phys. 2008; 70:385-390. [PubMed: 17869017]

22. Moreno-Jimenez M, Aristu J, Lopez-Picazo JM, et al. Dosimetric analysis of the patterns of local failure observed in patients with locally advanced non-small cell lung cancer treated with neoadjuvant chemotherapy and concurrent conformal (3D-CRT) chemoradiation. Radiother Oncol. 2008; 88:342-350. [PubMed: 18558448]

23. Stinchcombe TE, Morris DE, Moore DT, et al. Post-chemotherapy gross tumor volume is predictive of survival in patients with stage III non-small cell lung cancer treated with combined modality therapy. Lung Cancer. 2006; 52:67-74. [PubMed: 16499996]

24. Arrieta $\mathrm{O}$, Gallardo-Rincon D, Villarreal-Garza C, et al. High frequency of radiation pneumonitis in patients with locally advanced non-small cell lung cancer treated with concurrent radiotherapy and gemcitabine after induction with gemcitabine and carboplatin. J Thorac Oncol. 2009; 4:845852. [PubMed: 19487963]

25. Choy H, Jain AK, Moughan J, et al. RTOG 0017: a phase I trial of concurrent gemcitabine/ carboplatin or gemcitabine/paclitaxel and radiation therapy ("pingpong trial") followed by adjuvant chemotherapy for patients with favorable prognosis inoperable stage IIIA/B non-small cell lung cancer. J Thorac Oncol. 2009; 4:80-86. [PubMed: 19096311]

26. Blackstock AW, Ho C, Butler J, et al. Phase Ia/Ib chemo-radiation trial of gemcitabine and doseescalated thoracic radiation in patients with stage III A/B non-small cell lung cancer. J Thorac Oncol. 2006; 1:434-440. [PubMed: 17409896] 


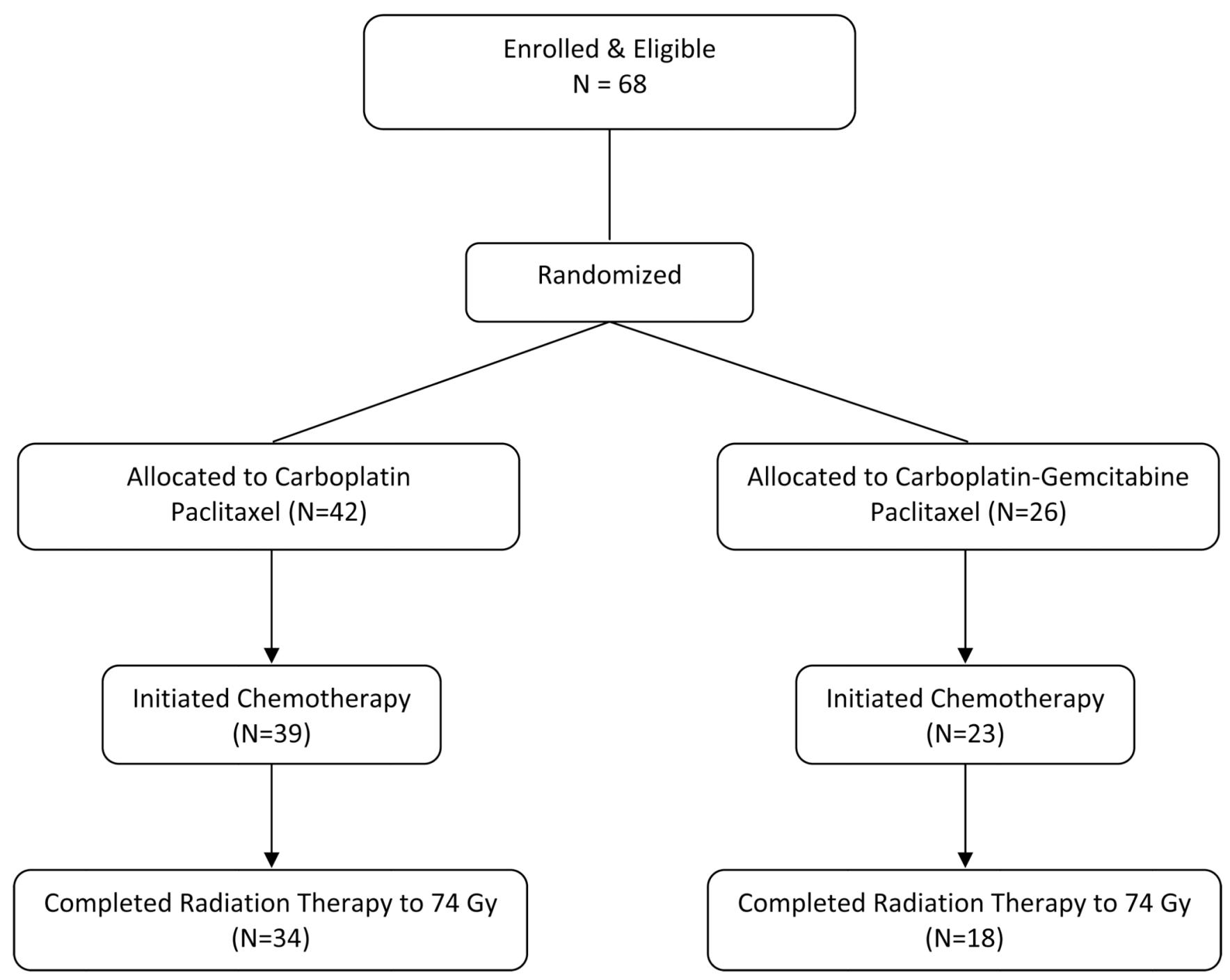

Figure 1.

Consort Diagram 
Table 1

Patient Demographic and Clinical Characteristics

\begin{tabular}{|c|c|c|}
\hline $\mathbf{N}(\%)$ & $\begin{array}{r}\operatorname{Arm~A} \\
N=42\end{array}$ & $\begin{array}{l}\operatorname{Arm} B \\
N=26\end{array}$ \\
\hline \multicolumn{3}{|l|}{ Gender } \\
\hline Male & $31(74)$ & $20(77)$ \\
\hline Female & $11(26)$ & $6(23)$ \\
\hline \multicolumn{3}{|l|}{ Age } \\
\hline median(range) & $62(44,76)$ & $58(38,79)$ \\
\hline \multicolumn{3}{|l|}{ Race } \\
\hline White & $38(90)$ & $22(85)$ \\
\hline Black & $4(10)$ & $4(15)$ \\
\hline \multicolumn{3}{|l|}{ Initial Diagnosis } \\
\hline Adenocarcinoma & $17(40)$ & $7(27)$ \\
\hline Squamous & $15(36)$ & $9(35)$ \\
\hline Undiff large & $2(5)$ & $1(3)$ \\
\hline Undiff non-small & $8(19)$ & $9(35)$ \\
\hline \multicolumn{3}{|l|}{ PS } \\
\hline 0 & $20(48)$ & $10(38)$ \\
\hline 1 & $22(52)$ & $16(62)$ \\
\hline \multicolumn{3}{|l|}{ Stage } \\
\hline IIIA & $26(62)$ & $10(38)$ \\
\hline IIIB & $16(38)$ & $16(62)$ \\
\hline \multicolumn{3}{|l|}{ Tumor Location * } \\
\hline Left side & $15(38)$ & $10(43)$ \\
\hline Right side & $24(62)$ & $13(57)$ \\
\hline \multicolumn{3}{|l|}{ Maximum Tumor Size } \\
\hline median (range) $(\mathrm{cm})$ & $4.7(1.0,9.2)$ & $6.6(1.8,10.0)$ \\
\hline
\end{tabular}

Abbreviations: Undiff=undifferentiated

3 patients data missing from each arm 


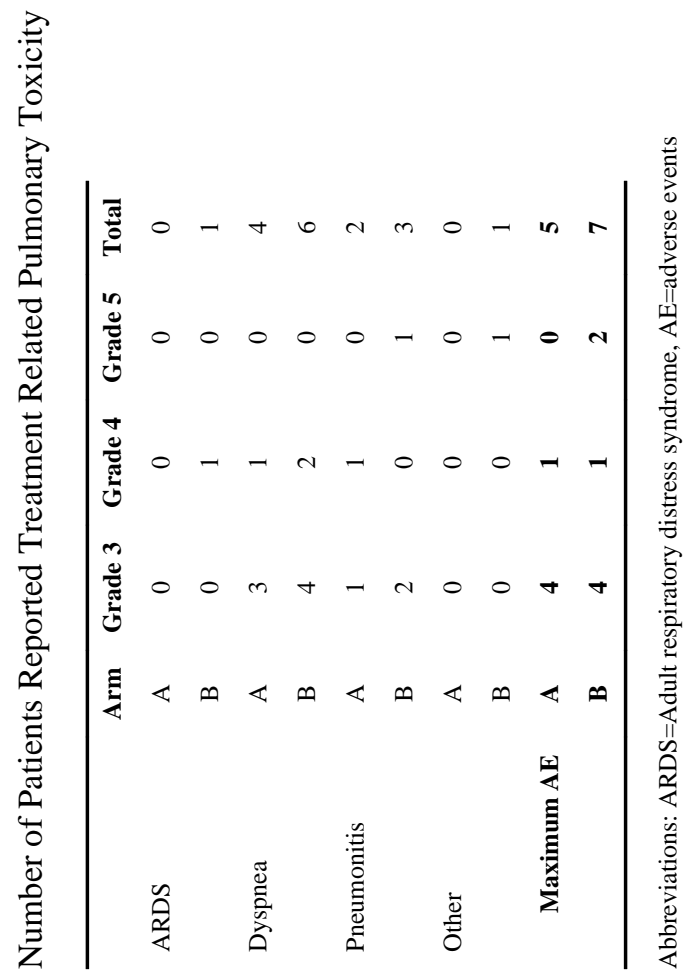

Int J Radiat Oncol Biol Phys. Author manuscript; available in PMC 2012 November 15. 
Table 3

Univariate Analysis on Pulmonary Toxicity - Wilcoxon 2-sided Test on Continuous Variables

\begin{tabular}{|c|c|c|c|c|}
\hline Median & Grade 0 - 2 & Grade 3 & Grade 4-5 & P-value * \\
\hline Age & 59 & 66 & 72 & 0.0047 \\
\hline T Stage & 2.5 & 2.5 & 2.0 & 0.7912 \\
\hline N Stage & 2.0 & 2.0 & 2.5 & 0.0343 \\
\hline Maximum Tumor Size & 5.2 & 5.3 & 6.4 & 0.6688 \\
\hline FEV-1 & 2.1 & 2.0 & 1.9 & 0.3508 \\
\hline TLV & 3387 & 4397 & 3098 & 0.9599 \\
\hline GTV1 & 111 & 115 & 271 & 0.2022 \\
\hline PTV1 & 772 & 725 & 1688 & 0.0946 \\
\hline TLV / GTV1 & 29.7 & 32.1 & 11.7 & 0.1133 \\
\hline TLV / PTV1 & 4.0 & 4.9 & 1.5 & 0.0639 \\
\hline V5 & 51 & 50 & 76 & 0.3875 \\
\hline V10 & 40 & 44 & 56 & 0.2536 \\
\hline V20 & 32 & 40 & 40 & 0.0168 \\
\hline Mean of Lung Dose & 1846 & 2185 & 1945 & 0.0973 \\
\hline Maximum of Lung Dose & 7966 & 7742 & 7881 & 0.4012 \\
\hline EQS & 12.7 & 13.4 & 12.5 & 0.6325 \\
\hline
\end{tabular}

* ${ }^{*}$-values are from Wilcoxon 2-sided test on pulmonary toxicity of grade $0-2$ vs. grade 3-5.

Abbreviations: FEV-1=Forced expiratory volume in1 second, GTV=Gross Tumor Volume, TLV=total lung volume, PTV=planning target volume, volume of tumor free lung receiving 5 (V5), 10 (V10), 20 (V20). T=Tumor, N=nodal, EQS=Equivalent square a measure of radiation field size. 
Table 4

Logistic Regression Statistics of Pulmonary Toxicity of Grade 0-2 vs. 3-5

\begin{tabular}{lrr}
\hline Variable & Odds Ratio (95\% CI) & Chi-square P-value \\
\hline Arm A vs. B & $17(1-301)$ & 0.0502 \\
N-stage & $0.11(0.01-1.48)$ & 0.0953 \\
V20 & $0.8(0.7-1.0)$ & 0.0414 \\
\hline
\end{tabular}

$\mathrm{N}$-stage (N0-N3), and V20 are continuous variables 
Table 5

Fisher's Exact Test of Pulmonary Toxicity and the 'Risk Factor'

\begin{tabular}{lrrc}
\hline Risk Factor & Grade 0-2 & Grade 3-5 & P-value \\
\hline N $0-2$ and V20 $<38$ (low risk) & 26 & 2 & 0.0313 \\
N3 or V20 $\geq 38$ (high risk) & 16 & 8 & \\
\hline
\end{tabular}

